Conclusion High reticulocyte count is associated with increased risk of death in patients awaiting LT. Remodelling UKELD to include high reticulocyte count improved accuracy of predicting death on the LT waiting list.

Competing interests None declared.

\section{PTU-064 PRE-LIVER TRANSPLANT BIOPSY IN HEPATOCELLULAR CARCINOMA: A POTENTIAL EXCLUSION CRITERION FOR TRANSPLANTATION?}

doi:10.1136/gutjnl-2012-302514c.64

${ }^{1} \mathrm{R}$ Young, ${ }^{*} \mathrm{M}$ Aldiwani, ${ }^{1} \mathrm{~A}$ Hakeem, ${ }^{1} \mathrm{~A}$ Nair, ${ }^{2} \mathrm{~J}$ Wyatt, ${ }^{1} \mathrm{G}$ Toogood, ${ }^{1} \mathrm{P}$ Lodge, ${ }^{3} \mathrm{R}$ Jones, ${ }^{1} \mathrm{R}$ Prasad. ${ }^{1}$ Department of Hepatobiliary and Transplant Surgery, St James's University Hospital, Leeds, UK; ${ }^{2}$ Department of Histopathology, St James's University Hospital, Leeds, UK; ${ }^{3}$ Department of Hepatology, St James's University Hospital, Leeds, UK

Introduction In cirrhotic patients with hepatocellular carcinoma (HCC) pre-liver transplant (LT) staging biopsy of the largest tumour is undertaken in some centres. Proponents advocate that poor differentiation confers such poor prognostic significance that it can be used as an exclusion criterion for LT, resigning patients to palliative treatments. We do not carry out staging biopsies and sought to interrogate its potential use and impact on our practice in the context of ever increasing demands for organs.

Methods 65 consecutive patients undergoing orthotopic LT for radiologically diagnosed HCC at St James's University Hospital between 2006 and 2011 were identified for analysis from a prospectively maintained database. All patients had cirrhosis and incidental tumours were excluded. Diagnosis was in accordance to published guidelines and various clinic-pathological parameters were recorded. MRI findings were correlated with explant histological examination. Median follow-up was 24 months. Student t test, Mann-Whitney U test or related samples Wilcoxon Signed Rank tests were used where appropriate. The Kaplan-Meier method was used to determine survival with Log-Rank and Cox stepwise regression for survival comparisons. $\mathrm{p}<0.05$ was considered to be statistically significant.

Results 3 year survival was $81 \%$ with the only independent predictor microvascular invasion $(p=0.019)$. In $5(7.7 \%)$ patients there was no HCC in the explant. A discrepancy between the definition of the largest lesion on pre-LT radiology and the largest explant tumour occurred in $5(7.7 \%)$ cases. Tumours were classified as well, moderately or poorly differentiated in 39 (30.2\%), 66 $(51.2 \%)$ and $24(18.6 \%)$ cases. In patients with multifocal HCC, 9 $(34.6 \%)$ had tumours of differing grades. In two $(7.7 \%)$ patients the largest tumour was well differentiated while smaller tumours in the explant were poorly differentiated. In one patient the largest lesion was benign with other smaller invasive carcinomas confirmed histologically.

Conclusion There is a need to optimise LT selection strategies for HCC. Microvascular invasion was the only independent predictor of outcome and the challenge of predicting it pre-operatively remains. Crucially, the largest lesion was not always representative of overall tumour burden or biological aggression and its potential use to exclude patients from curative treatment is questionable.

Competing interests None declared.

\section{PTU-065 LONG TERM OUTCOMES OF LIVER TRANSPLANTATION FOR WILSON'S DISEASE: A SINGLE CENTRE EXPERIENCE}

doi:10.1136/gutjnl-2012-302514c.65

H Al-Hilou, S Hebbar, ${ }^{*}$ B K Gunson, L C Claridge, W-K Syn, A P Holt. Liver unit, University Hospitals Birmingham NHS Foundation Trust, Birmingham, UK

Introduction Wilson's disease is an autosomal recessive disorder of copper metabolism which may manifest as acute or chronic liver disease and/or neuropsychiatric disorders. Orthotopic liver transplantation (OLT) is life saving when performed for acute liver failure or end-stage liver disease but there are conflicting reports on the effects of OLT on neuropsychiatric symptoms. Furthermore, a recent report from an Italian group has highlighted poor outcomes following OLT in those with neuropsychiatric symptoms.

Aim To evaluate the indications and outcomes of OLT for patients with Wilson's disease and in particular to assess the effects of OLT on neuropsychiatric symptoms, and whether these disorders are associated with poor outcomes.

Methods Data were acquired by retrospective analysis of case notes and databases.

Results 21 adults with Wilson's disease have been transplanted in Birmingham between 1987 and 2011. $53.3 \%$ (11/21) were performed for acute liver failure and $42.8 \%$ (9/21) for decompensated cirrhosis. One patient was transplanted due to hepatocellular carcinoma. Median age was 19.5 at diagnosis and 25 at time of OLT. 10 patients had neuropsychiatric symptoms prior to OLT (Parkinsonism in three patients, anxiety in two patients, epilepsy, OCD, depression, ataxia and migraine). These symptoms resolved in $4 / 10$ post-OLT. However, four patients developed new onset neuropsychiatric symptoms within 2 weeks of OLT; two patients had seizures, one developed psychosis and another became euphoric. Kayser-Fleisher (KF) rings are purportedly associated with neuropsychiatric disorders; in our cohort $61.9 \%(13 / 21)$ of patients had KF rings and $11 / 13$ of these had neuropsychiatric symptoms. Four patients had significant post-operative complications comprising four haemorrhagic episodes and two biliary leaks. Actuarial survival rates were $95 \%$ at both 1 and 5 years. One patient died within 3 months of OLT and three patients died $>10$ years post OLT. 3/4 deaths were due to multi-organ failure secondary to sepsis. Graft survival was $90 \%$ at 1 year and $81 \%$ at 5 years. Five patients have required re-grafting; three due to chronic rejection and two due to hepatic artery thrombosis. There were no appreciable differences in long term outcomes between those with and without pre-existing neuropsychiatric symptoms; $2 / 4$ deaths and 2/5 re-grafts were in those with neuropsychiatric manifestations.

Conclusion Outcomes of OLT for Wilson's disease are comparable to transplantation for other diagnoses. Neuropsychiatric symptoms improved in $<50 \%$ of patients post-OLT and may also occur for the first time following OLT. At our centre pre-existing neuropsychiatric symptoms were not associated with poor outcomes.

Competing interests None declared.

\section{PTU-066 AN "ALCOHOL CONTRACT" HAS NOT REDUCED RATES OF POST-TRANSPLANT DRINKING FOLLOWING TRANSPLANTATION FOR ALCOHOLIC LIVER DISEASE}

doi:10.1136/gutjnl-2012-302514c.66

S Kendrick, ${ }^{*}$ A Elsharkawy, S Latimer, M Hudson, S Masson. Liver Unit, Newcastle upon Tyne Hospitals Foundation Trust, Newcastle upon Tyne, UK

Introduction Reports of patients returning to drinking after orthotopic liver transplantation (OLT) for alcoholic liver disease (ALD) remain a source of unease for professionals and the public. Recidivism rates of $10 \%-16 \%$ are reported with a low rate of alcoholrelated graft loss. ${ }^{12}$ In 2005, the UK Transplant liver advisory group recommended an "alcohol contract" in which ALD patients listed for OLT confirmed in writing their commitment to abstinence. ${ }^{3}$ The purpose of our study was to measure the rates and consequences of post-OLT alcohol intake in a UK transplant program and assess the effect of the "alcohol contract" on rates of post-transplant drinking. Methods Prospectively collected data were reviewed for 100 randomly selected patients transplanted for ALD-32 patients 